Classification

Physics Abstracts

$71.60-25.00$

\title{
Plasticity of a silicon bicrystal: a HREM study
}

\author{
Jany Thibault $\left({ }^{1}\right)$, Jean-Luc Putaux $\left({ }^{1}\right)$, Alain Jacques $\left({ }^{2}\right)$, Amand George $\left({ }^{2}\right)$ \\ and Mohamed Elkajbaji $\left({ }^{3}\right)$
}
( $\left.{ }^{1}\right)$ Département de Recherche Fondamentale, SPh/S, Centre d'Etudes Nucléaires, 85X, 38041 Grenoble, France
$\left({ }^{2}\right)$ Laboratoire de Physique du Solide, URA155, Ecole des Mines, Parc de Saurupt, 54042 Nancy, France
$\left({ }^{3}\right)$ Département de Physique, Faculté des Sciences, BP38/S, Agadir, Marrocco

(Received October 01, 1990; accepted January 25, 1990)

\begin{abstract}
Résumé. - Le bicristal de silicium contenant le joint de flexion $\Sigma=9(\overline{1} 2 \overline{2})$ [011] est utilisé comme matériau modèle pour étudier, à l'aide de la microscopie électronique à haute résolution, les mécanismes de déformation à l'échelle atomique. Les mystères des tout premiers stades de déformation du joint ont été élucidés. On a montré que les dislocations créés dans les grains par la contrainte extérieure s'incorporaient au joint par glissement et par montée. L'étude de l'évolution de la structure du joint de grains a été menée pour différentes conditions de déformation et de température. L'accumulation de dislocations DSC le long du joint entraîne une variation de l'angle de desorientation entre les grains; en compression l'angle augmente et différents $\Sigma$ sont balayés lors de la déformation. Les points importants sont que l'évolution du joint peut se décrire à l'aide d'un nombre limité d'unités structurales et que, pour un même $\Sigma$, différentes structures sont obtenues en fonction de la température de déformation.
\end{abstract}

\begin{abstract}
A perfect silicon bicrystal containing only one pure tilt grain boundary (GB) $\Sigma=9$ $(\overline{1} 2 \overline{2})[011]$ is used as a model in order to study the very early stages of the mechanisms occurring at atomic scale close to the GB. The high resolution electron microscopy observations showed that most dislocations induced by the deformation integrated the GB by glide and climb. The evolution of the structure of the original GB has been studied for different strains and at different temperatures. The accumulation of the DSC dislocations into the GB leads to a variation in the misorientation angle between the two grains. In compression it increases, whereas it decreases in tension. Consequently, various $\Sigma$ GBs have been detected. The key points are that, firstly, the structural evolution of the GB can be described with a limited number of structural units and secondly, depending on the deformation temperature, one $\Sigma$ GB can exhibit two different structures.
\end{abstract}

\section{Introduction.}

The high resolution electron microscopy (HREM) has been widely used to observe the structure of grain boundaries in semiconductors. In this paper, we will show that this technique is a powerful 
method to study the very early stages of the mechanisms occurring during the evolution of the materials under different constraints: stresses, temperature... This paper will report the main results obtained in a simple case: the deformation of a pure tilt silicon bicrystal. The study of this model case has permitted to achieve a better knowledge of the mechanisms taking place at atomic level during the deformation. The original sample was a perfect bicrystal with the $\Sigma=9(\overline{1} 2 \overline{2})[011]$ tilt grain boundary (GB). The tilt axis is [011] common to both grains, the misorientation angle is $38^{\circ} 94$ and the GB plane is $(\overline{12} \overline{2})_{\mathrm{I}}$ parallel to $(12 \overline{2})_{\mathrm{II}}$ (index I and II refer to grain I and II); the GB structure has a mirror glide symmetry [1]. The atomic structure $[2,3]$ is made up with one type of structural unit (SU) called $M$ (a five-seven atom ring [4]). However, the sequence of one GB period is $\mathrm{M}^{-} \mathrm{M}^{+}$where $\mathrm{M}^{-}$and $\mathrm{M}^{+}$are mirror related. After deformation, the samples were cut perpendicularly to the [011] axis and observed by HREM down to the same axis. The HR pictures have been recorded in a JEOL 200CX TEM $(C s=1 \mathrm{~mm})$. The use of a higher voltage leads to radiation damages in the silicon crystal.

\section{Deformation mechanisms.}

The study of the deformation has been previously performed with in-situ $1 \mathrm{MeV}$ electron microscopy and Xrays synchrotron topography [5-7]. These steps allowed to have a detailed view of the mechanisms at a mesoscopic scale which cannot be achieved by HREM observations and to determine the best HREM experiments needed to clarify mechanisms at atomic scale.

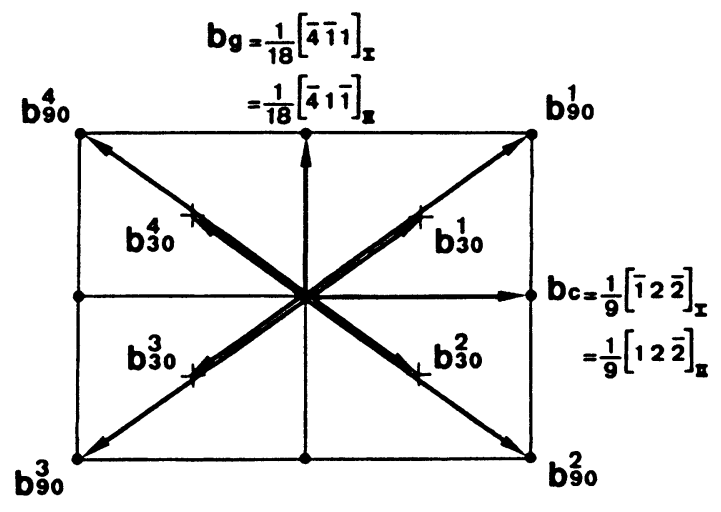

Fig. 1. - Projection along [011] of the DSC lattice of the $\Sigma=9 \mathrm{~GB}$. The lattice is a body centered lattice and is based on the three perpendicular unit vectors: $1 / 9[\overline{1} 2 \overline{2}]_{\mathrm{I}}, 1 / 18[\overline{4} \overline{1} 1]_{\mathrm{I}}$ and $1 / 2[011]_{\mathrm{I}}$ (I refers to grain I). Various vectors used in the text are indicated.

The stress axis was contained in the GB plane, common to both grains. It was chosen in such a way that the main activated slip system and the additional one were suitable for HREM observations: the strain induced dislocations were aligned along the [011] common axis. Details on the induced slip systems can be found elsewhere [8]. The dislocations were mainly $60^{\circ}$ dislocations dissociated in two Shockley partials. Dissociated screw dislocations belonging to the bicrystal common slip system were also found. Pile-ups of dislocations formed towards the GB which acts generally as a strong obstacle even if the transmission of dislocations which do not belong to the common slip system has been observed in specific deformation conditions [9]. 
The main point we wanted to illustrate is that the stress at the head of the pile-up is generally relieved by the entrance and the decomposition of the bulk dislocations into the GB. The absorption occurs by the glide and climb of the residual grain boundary dislocations (GBDs). In the following the mechanisms will be described with grain I as the reference. The projection along [011] of the $\Sigma=9$ DSC lattice is shown in figure 1, the different vectors used in the following to describe the dislocations are indicated.

\subsection{INDIVIDUAL DISLOCATION AND GB INTERACTION.}

2.1.1 $60^{\circ}$ dislocation and $G B$ interaction. - One has to mention, as demonstrated in [10], provided the $\mathrm{GB}$ is of $\Sigma=3 \mathrm{~N}$ type, the Burgers vector (Bv) of the partials on one of the slip systems belongs necessarily to the DSC lattice. In our cas, the Bv of the partials on the primary slip planes $(11 \overline{1})_{\mathrm{I}}$ and $(1 \overline{1} 1)_{\mathrm{II}}$ does exhibit this property, unlike the Bv of the partials on the addtional planes $(1 \overline{11})_{\mathrm{I}}$ and $(11 \overline{1})_{\mathrm{II}}$. In the compression tests, the $90^{\circ}$ partial of the $60^{\circ} \mathrm{D}$ is the head partial and consequently touches first the GB. The $90^{\circ}$ partial arriving on the primary slip plane $(11 \overline{1})_{\mathrm{I}}$ is a DSC dislocation but not an elementary DSC dislocation. As shown in [8] it can and does decompose in two elementary DSC dislocations.

$$
b_{90}^{1}=b_{\mathrm{c}}+b_{\mathrm{g}}
$$

Although its BV is a DSC vector, $b_{\mathrm{c}}$ is not an ordinary GDB, since it is located at the intersection of the GB and the stacking fault connected to the $30^{\circ}$ trailing partial remaining in the bulk (Fig. 2a). It is also different from a Shockley partial. It is called an "imperfect GBD" in [11] even though it does not create any faulted area in the GB. The perfect GDB $b_{\mathrm{g}}$ is glissile into the $\Sigma=9 \mathrm{~GB}$ and moves away from the impact point under internal stresses. The repulsion between the dislocations at both ends of the SF is annihilated and then under the applied and the pile-up stresses, the trailing partial enters the GB. This results in a final configuration decomposed by climb in two residual elementary GBDs: one perfect $b_{\mathrm{c}}$ GBD and one perfect $b_{30}^{1}$ GBD (Fig. 2b).

The $90^{\circ}$ partial dislocation arriving on the additional plane $(1 \overline{1} 1)_{\mathrm{I}}$ is not a DSC dislocation, nevertheless schemes of integration have been proposed [11] which all are assisted by the emission of two $b_{\mathrm{g}}$ glissile dislocations in the GB. This results in an isolated residue $b_{30}^{4}$ (Fig. 3).

2.1.2 Screw and GB interaction. - Theoretically, this is the simplest case. Like in the bulk, the two $30^{\circ}$ Shockley partials can recombine and by cross slip pass in the other grain. As mentionned in [11] the emission of $b_{\mathrm{g}}$ glissile GBDs from the dissociation of the recombined screw dislocation or from the $30^{\circ}$ partial touching the GB is highly improbable. However, if climb is available, this gives the possibility for the constricted screw dislocation to decompose in two $b_{30}$ DSC dislocations into the GB and consequently this impedes the transmission of the screw. In fact, because no SF is crystallographically necessary in the GB, the energy of this final structure is less than the one of a transmitted redissociated screw dislocation. Figure 4 shows the residual configuration stemming from such a high temperature mechanism $\left(T=1120^{\circ} \mathrm{C}\right)$. At low temperature $\left(T<950^{\circ} \mathrm{C}\right), \mathrm{HREM}$ never reveals such a configuration. Having no other choice, the screw dislocation traverses the GB without leaving any GB residual dislocation $[7,11]$.

2.2 GBDs INTERACTION. - As the GBDs can move in the GB, they can interact and this leads to new residues. Details of different interactions have been studied by HREM [8]. Only one example will be described here: the entrance in compression of two $60^{\circ} \mathrm{Ds}$ coming on the primary planes on both sides of the GB plane. This is the key point for the further evolution of the GB under higher strain. 

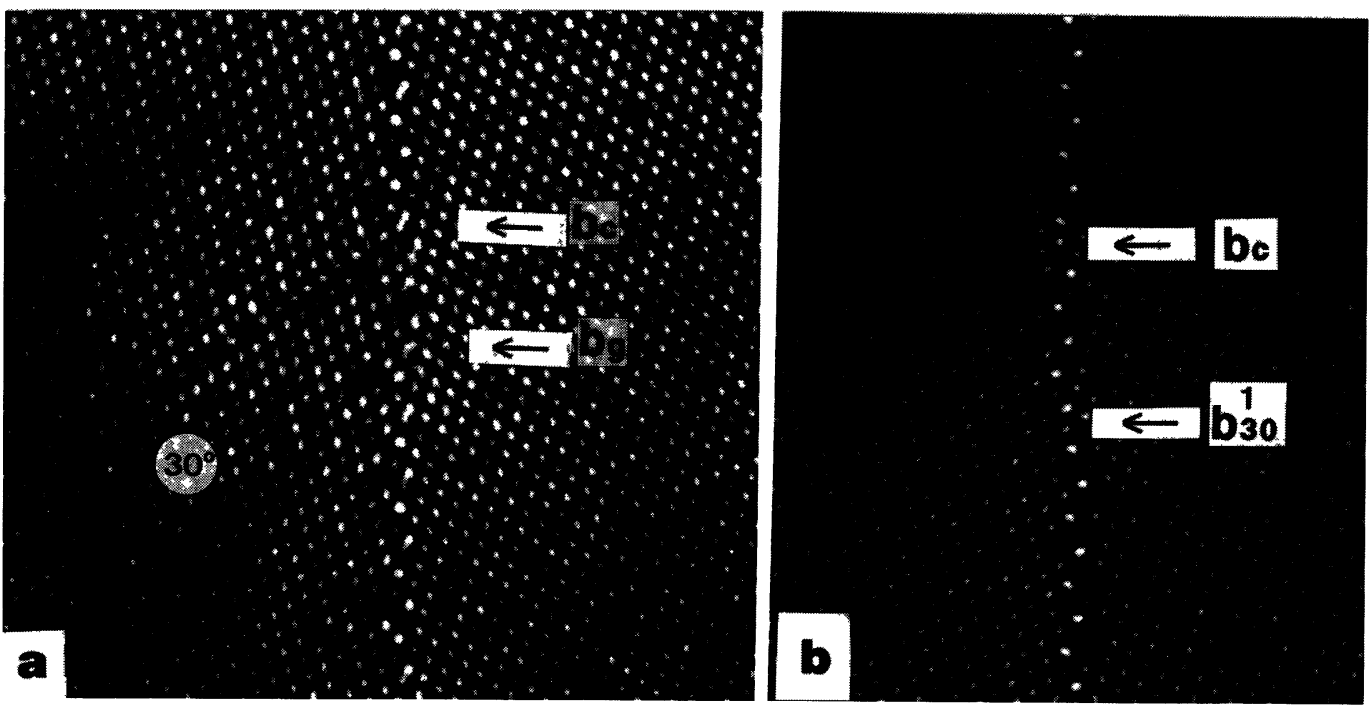

Fig. 2. - Entrance of the $60^{\circ}$ dislocation in compression on the (11̄i) I primary slip plane: a) one glissile $b_{\mathrm{g}}$ GBD is being emitted in the GB by the leading partial $\left.b_{90}^{1}, \mathrm{~b}\right)$ two residual GBDs are left by the complete entrance and decomposition of the incoming dislocation.

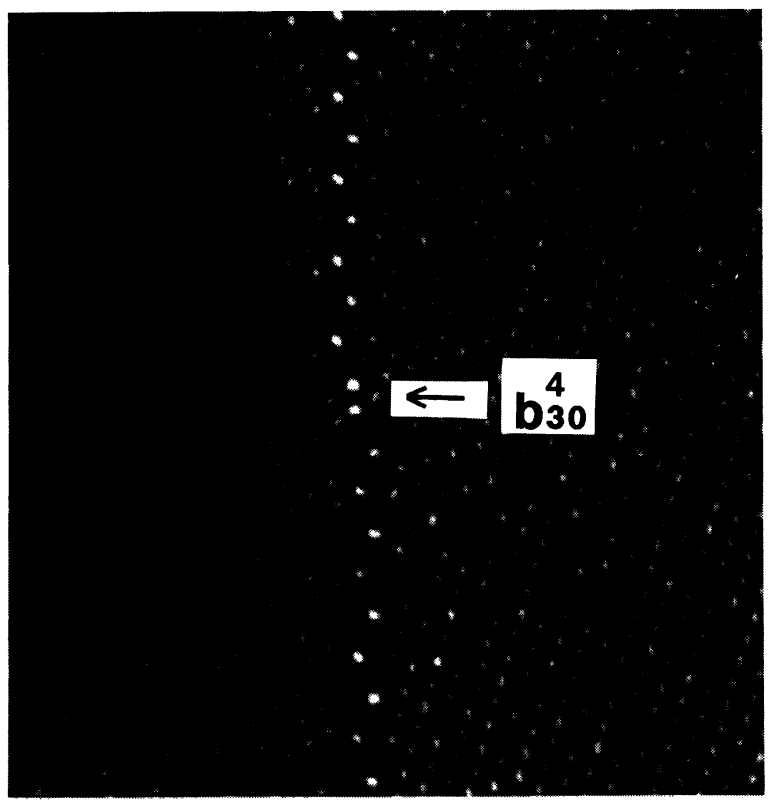

Fig. 3. - Residual dislocation $b_{30}^{4}$ resulting from the entrance and the decomposition of a $60^{\circ} \mathrm{D}$ on the $(1 \overline{1} 1)_{\mathrm{I}}$ additional slip planes. Two $b_{\mathrm{g}}$ have been emitted. 
The entrance of a $60^{\circ} \mathrm{D}$ on $(11 \overline{1})_{\mathrm{I}}$ leads to three GBDs: $b_{\mathrm{c}}, b_{30}^{1}, b_{\mathrm{g}}$ whereas the entrance of the $60^{\circ} \mathrm{D}$ on the $(1 \overline{1} 1)_{\mathrm{II}}$ leads to the three GBDs: $b_{\mathrm{c}}, b_{30}^{2},-b_{\mathrm{g}}$. The screw components of the two $b_{30}$ have opposite sign. Generally, the two primary slip systems correspond in the two grains and consequently dislocations of one and the other grain arrive in the same area of the GB. Furthermore they are in attractive interaction. The two $b_{30}$ GBDs interact by climb, the driving force being the attractive force between these two $b_{30}$ : the result of the interaction of these two GBDs is $b_{c}$. The two glissile dislocations $b_{\mathrm{g}}$ annihilate. The net result is:

$$
b_{60}^{1}+b_{60}^{2}=b_{c}+b_{c}+b_{c}
$$

This resulting triplet configuration in shown in figure 5.

2.3 LOW DEFORMATION (1.7\%). - - As the strain increases, the number of dislocations entering the $\mathrm{GB}$ increases. In compressions, at a temperature less than $950^{\circ} \mathrm{C}$, different residual GBDs are found along the GB (Fig. 6a). At a higher temperature, only one type of residual GBD has been observed: the $b_{\mathrm{c}} \mathrm{GBD}$ resulting from glide and climb interaction of the previous residues as described in section 2.2. These $b_{c}$ are more or less homogeneously distributed along the GB, this results in an almost prefect sub-grain boundary superimposed to the original $\Sigma=9 \mathrm{~GB}$, (Fig. $6 \mathrm{~b}$ ). The defects found along the GB deformed in tension are different. At relatively low temperature a mixture of non elementary DSC dislocations and elementary GBDs $-b_{c}$ has been detected as explained in [11]. Nevertheless, the component perpendicular to the GB of the Burgers vector has a sign opposite to the one obtained in compression. This makes the misorientation angle between the two grains decrease whereas it increases in compression.

2.4 HIGH DEFORMATION (7\%). - At higher strain the number of elementary defects increases a lot all along the GB and in compression, the misorientation between the grains has been found to achieve a macroscopic angle of about $50^{\circ} 5$ corresponding to a $\Sigma=11(233) \mathrm{GB}$. Due to the inhomogeneity of the deformation along the GB, different $\Sigma$ GBs have been detected. $\Sigma=187$ (599) $\left(\theta=42^{\circ} 88\right)$ and $\Sigma=41$ (344) $\left(\theta=55^{\circ} 88\right)$ for instance have been described elsewhere [14]. In this paper, we will concentrate on the $\Sigma=11 \mathrm{~GB}$ induced by the deformation.

\section{Structural evolution of the $\Sigma=9 \mathrm{~GB}$.}

3.1 STRUCTURE OF THE GBDs. - The atomic structure of the elementary DSC dislocations has been determined by HREM [12-15]. The structure of the two elementary DSC dislocations $b_{\mathrm{c}}$ and $-b_{c}$ is presented in figure 7 . The comparison between the simulated images and the micrographs allows to say that the two dislocations are associated with two different structural units (SUs) [15].

The $b_{c}$ GBD is associated with the so-called "T" SU which is the basic unit of the mirror twin GB $\Sigma=3(11 \overline{1})[011]\left(\theta=70.4^{\circ}\right)$. It has to be noticed that the $b_{\mathrm{c}} \mathrm{SU}$ is always inserted between the two $\mathrm{M}^{-}$and $\mathrm{M}^{+}$SUs of the original $\Sigma=9 \mathrm{~GB}$. The presence of $b_{\mathrm{c}}$ can be written as $\mathrm{M}^{-} \mathrm{M}^{+} \mathrm{TM}^{-}$ $\mathrm{M}^{+}$.

On the other hand $-b_{c}$ is associated with a structural unit " $C$ ", which can be used to built the perfect Si crystal defined as the $\Sigma=1(01 \overline{1})$ [011] grain boundary with $\theta=0^{\circ}$. However, in this case the $\mathrm{C}$ unit is inserted in between the seven and the five atom ring of one $\mathrm{M}$ basic unit. As a matter of fact the structure of the original $\Sigma=9 \mathrm{~GB}$ can also be read as a stacking of " $L$ " SUs [16] as described in [17]. The presence of $-b_{c}$ can be translated by the sequence $\mathrm{L}^{-} \mathrm{L}^{+} \mathrm{CL}^{-} \mathrm{L}^{+}$ $\mathrm{L}^{-}$. This $\mathrm{L}$ unit corresponds to the core of the non dissociated Lomer edge dislocation which is in fact the defect accounted for a sub-grain boundary whose tilt axis is [011] and whose GB plane 


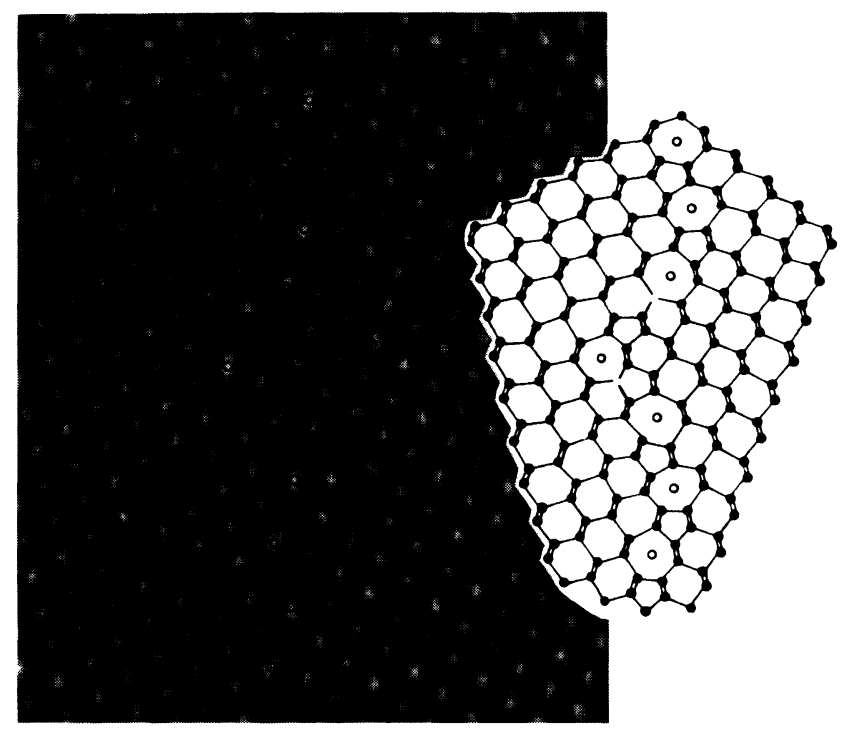

Fig. 4. - Result of the integration and the climb dissociation of a screw dislocation into the $\Sigma=9 \mathrm{~GB}$. Not yet Burgers vector (in projection) can be found around the global defect.

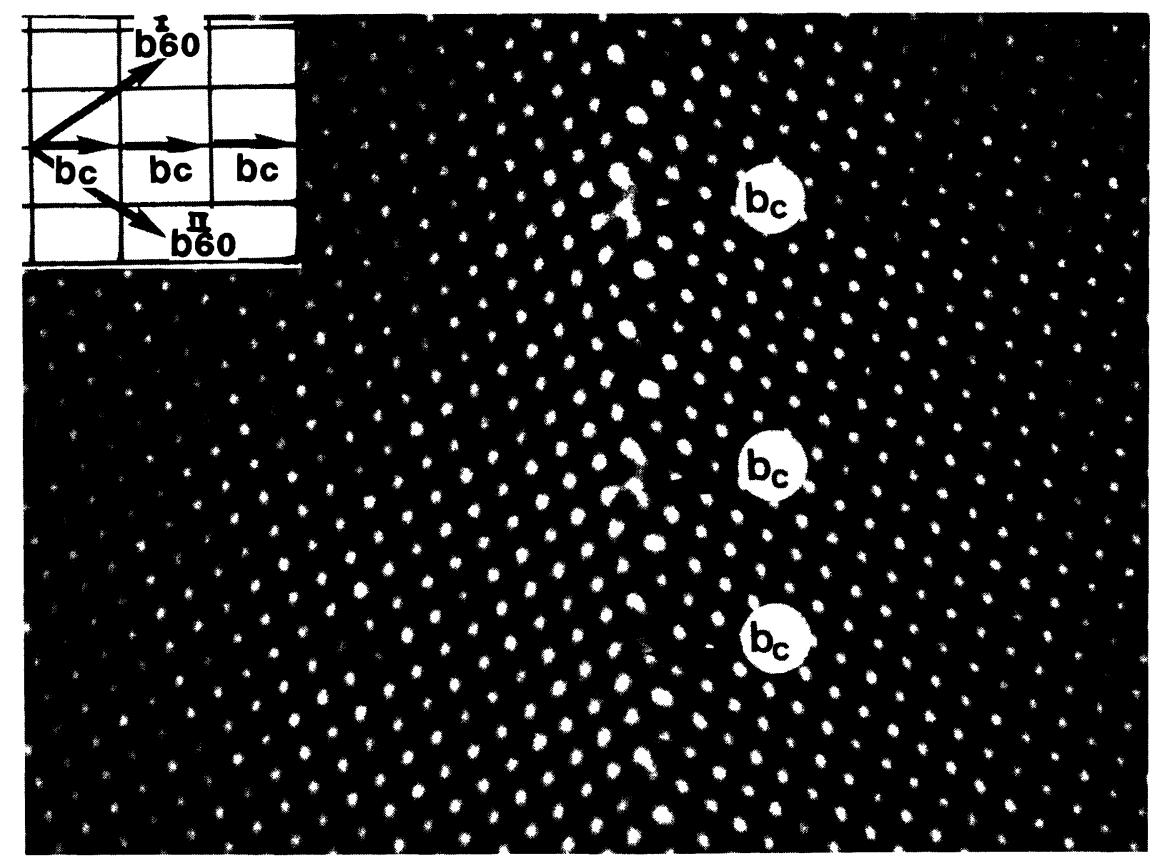

Fig. 5. - Final configuration resulting from the interaction in the GB of two $60^{\circ}$ dislocations coming from both adjacent grains. The $b_{c}$ triplet configuration is decomposed by climb. 


\section{$850^{\circ} \mathrm{C}-1.7 \%$}
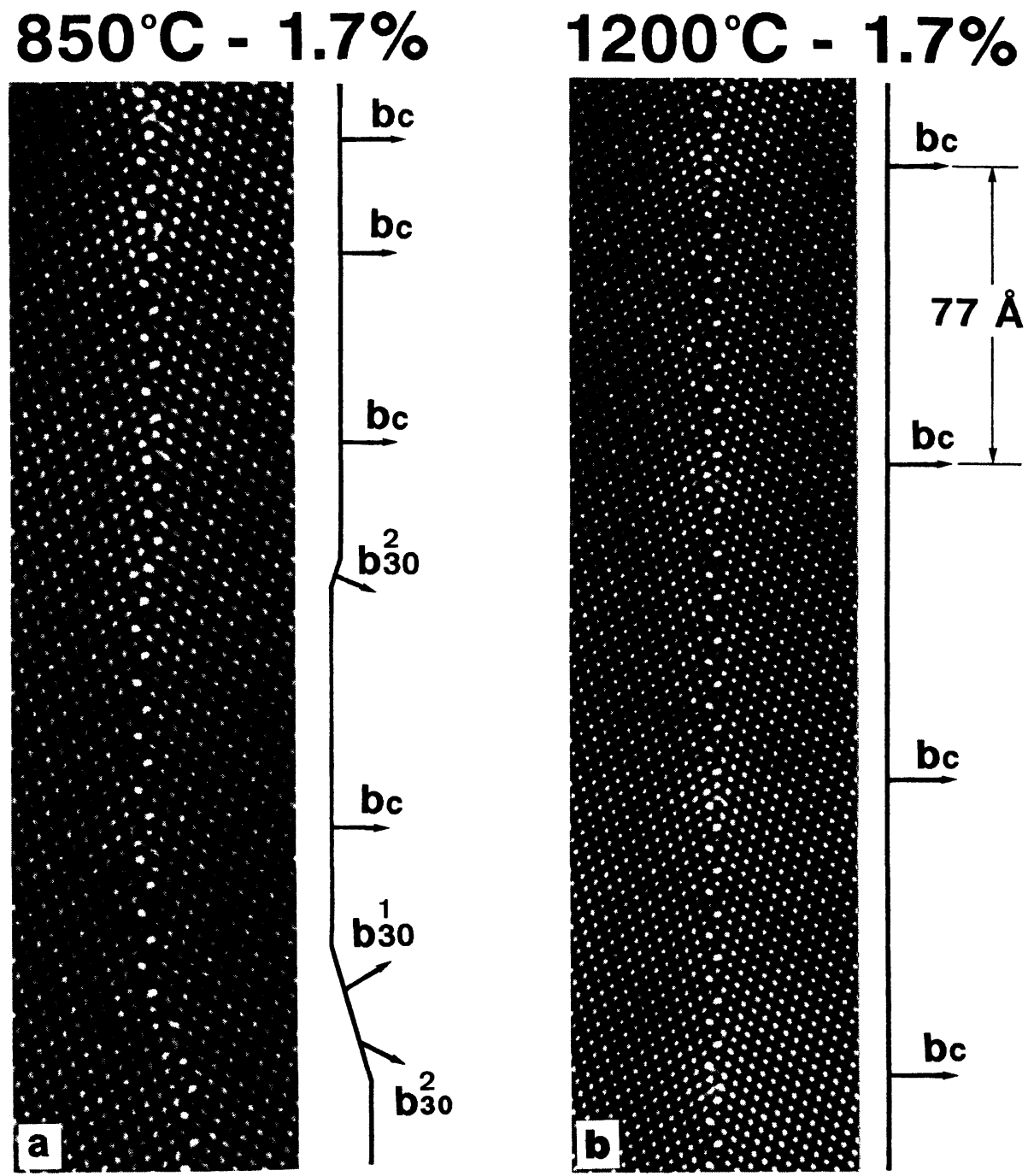

Fig. 6. - After deformation, more or less perfect sub-grain boundaries are superimposed to the original $\Sigma=9$. These distributions are obtained in compression and a) at $850^{\circ} \mathrm{C}, \mathrm{b}$ ) at $1200^{\circ} \mathrm{C}$. The b configuration is periodic.

is $(01 \overline{1})$. Thus, the evolution of the $\Sigma=9 \mathrm{~Gb}$ structure either in tension or in compression might be described the scheme in table $\mathrm{I}$.

3.2 STRUCTURE OF THE DEFORMATION INDUCED $\Sigma=11 \mathrm{~GB}$. - As the strain increases in compression, the extrinsic SUs associated with the $b_{c}$ 's accumulate more or less regularly along the GB owing to the climb of the GBDs. As a consequence, following the above scheme, different $\Sigma$ values are swept during the deformation. We will now describe the structures of the $\Sigma=11$ GB $\left(\theta=50.48^{\circ}\right)$ obtained under different deformation temperatures in compression.

Following the simplest scheme and accumulating $\mathrm{T}$ units along the $\mathrm{GB}$, the $\Sigma=11 \mathrm{~GB}$ is obtained when two $T$ units are introduced per one period of the original $\Sigma=9$. The sequence might 

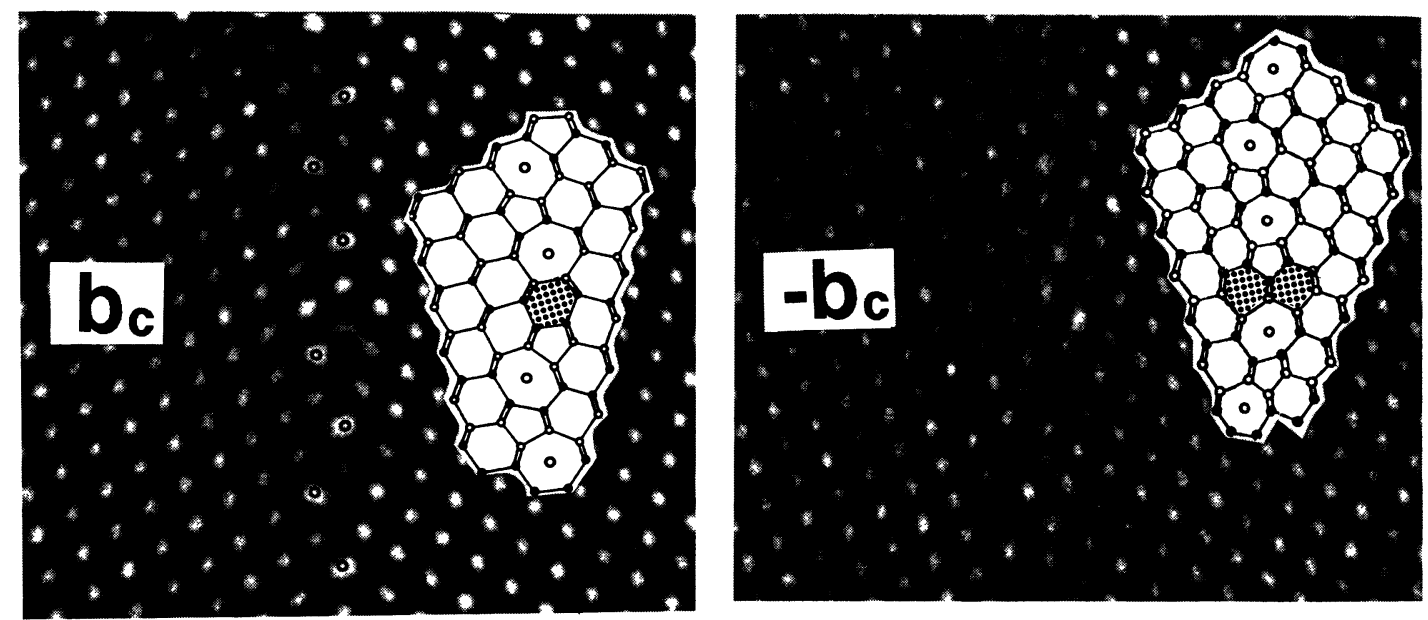

Fig. 7. - Structure of the two elementary GBDs $b_{\mathrm{c}}$ and $-b_{\mathbf{c}}$. The first is associated with the six-atom ring "T" found as the structural unit of the $\Sigma=3$ (111) favoured GB and the second is associated with a "C" structural unit which the perfect crystal (favoured $\Sigma=1(01 \overline{1}) \mathrm{GB}$ ) can be built with.

Table I. - Schematic evolution of the original $\Sigma=9 G B$ depending on the deformation condition (tension or compression). The original $\Sigma=9$ can be described either with the $L$ units or with the $M$ units. In tension the addition of $C$ units (associated with the $\Sigma=9 D S C$ dislocation $-b_{c}$ ) would lead to a decrease in the misorientation angle and consequently to the appearance of $\Sigma=1 \mathrm{~GB}$. In compression the addition of $T$ units (associated with $b_{c} ; \Sigma=9$ as reference) would lead to the $\Sigma=3$ twin $G B$.

\begin{tabular}{|c|c|c|c|c|}
\hline $\begin{array}{l}\Sigma=1 \\
\theta=0\end{array}$ & $\begin{array}{c}\mathrm{C} \text { unit } \\
<---- \\
\text { tension }\end{array}$ & $\begin{array}{c}\text { two descriptions of } \\
\Sigma=9 \\
\theta=38^{\circ} 94\end{array}$ & $\begin{array}{c}\text { T unit } \\
----> \\
\text { compression }\end{array}$ & $\begin{array}{c}\Sigma=3 \\
\theta=70^{\circ} 3\end{array}$ \\
\hline \multirow{3}{*}{ C } & \multirow{3}{*}{$\mathrm{L}^{-}$} & \multirow{2}{*}{$\mathrm{L}^{-} \mathrm{M}^{-}$} & $\mathbf{M}^{-}$ & $\mathrm{T}$ \\
\hline & & & & \\
\hline & & \multirow{2}{*}{$\mathrm{L}^{+}$} & $\mathrm{M}^{+}$ & $\mathrm{T}$ \\
\hline C & $\mathrm{L}^{+}$ & & & \\
\hline \multirow{2}{*}{ C } & \multirow{2}{*}{ C } & $\mathrm{L}^{-}{ }^{\mathbf{M}}$ & $\mathrm{T}$ & $\mathrm{T}$ \\
\hline & & \multirow{3}{*}{$\mathrm{L}^{+}$} & $\mathbf{M}^{-}$ & $\mathrm{T}$ \\
\hline \multirow[t]{2}{*}{ C } & $\mathrm{L}^{-}$ & & & \\
\hline & $\mathrm{L}^{+}$ & & $\mathbf{M}^{+}$ & $\mathrm{T}$ \\
\hline
\end{tabular}

be written: $\left(\mathrm{M}^{+} \mathrm{TM}^{-} \mathrm{T}\right)$. in fact, we did find this structure in specimens deformed at temperatures less than $950^{\circ} \mathrm{C}$ (Fig. 8a). This structure preserves the symmetry of the original GB i.e. the mirror glide symmetry. However, the basic SUs are highly distorded by shear and even though the structure looks the simplest, that certainly costs in energy.

At a higher deformation temperature, the final structure was different and a new SU must be introduced in order to describe the $\Sigma=11 \mathrm{~GB}$ properly (Fig. $8 \mathrm{~b}$ ). This SU can be found in the core of the GBD $b_{90}^{1}=b_{\mathrm{c}}+b_{\mathrm{g}}[14]$. It is made with a seven-five atom ring as shown in figure 7 but 

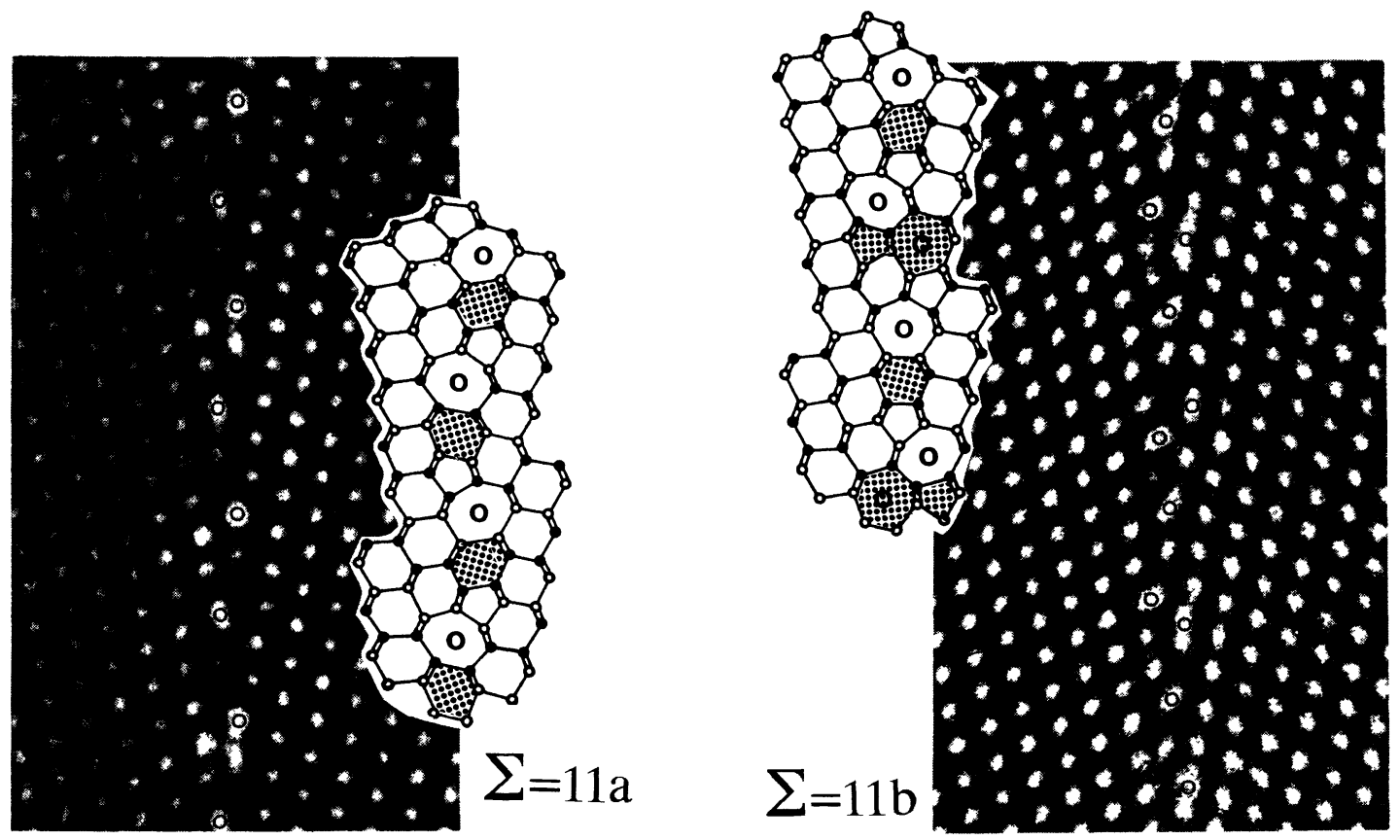

Fig. 8. - The two structures of the $\Sigma=11 \mathrm{~GB}$ stemming from a strain a) at $950^{\circ} \mathrm{C}$ and b) at $1200^{\circ} \mathrm{C}$. The low temperature configuration is based on the accumulation of $\mathrm{T}$ units and has a mirror glide symmetry whereas the b) structure contains the new P unit and has a mirror symmetry.

differs from the other seven-five atom rings $L$ or $M$ by the way the seven atom ring and the five atom ring are connected. The period of the GB is now given by the sequence: $\left(\mathrm{M}^{+} \mathrm{TM}^{-} \mathrm{P}^{+} \mathrm{M}^{-}\right.$ $\left.\mathrm{TM}^{+} \mathrm{P}^{-}\right)$. It is twice the period of the low temperature induced $\Sigma=11 \mathrm{~GB}$. This structure was also detected by Bourret et al. [17] in as-grown Ge bicrystals.

The two structures exhibit very close static energies. As a matter of fact, one could built the $\Sigma=3$ GB with this "P" $\mathrm{SU}$, giving rise to a glide mirror structure $\left(\mathrm{P}^{+} \mathrm{P}^{-}\right)$with a high static energy [14].

\section{Conclusions.}

Two main points have to be emphasized: one on the mechanisms, the other one on the structural evolution occurring during deformation.

Firstly, the $\Sigma=9$ is generally a strong obstacle to the transmission of the glide. Owing to the possibility of emitting gliding GBDs, the deformation induced $60^{\circ}$ dislocations are stopped by the GB where they decompose in elementary GBDs. The screw dislocations belonging to the common slip system traverse the GB by a cross-slip mechanism. Moreover, if climb is possible, the transmission becomes more unlikely.

Secondly, the evolution of the GB structure under stresses at different temperatures can be described with a small number of SUs which can be related to the concept of "favoured" GBs [18]: $\Sigma=1, \Sigma=9$ and $\Sigma=3$ tilt GBs. However some structures exhibit "non favoured" SUs such as the P units. In fact, from the point of view of the SU content, $\Sigma=11 \mathrm{~GB}$ is just in between the two favoured GBs $\Sigma=9$ and $\Sigma=3$. In [18], the authors claims that the translation states of 
the two delimitating GBs must be similar to avoid compatibility stresses. In our case the two delimitating GBs have different translation states: $\Sigma=9$ has a mirror glide symmetry and $\Sigma=3$, a pure mirror symmetry. Thus a mixture of the units of both GBs introduces a shear of each unit. The appearance of a third type of unit at high temperature leads to a different distribution, of the shear of each units and might help to stabilize the GB structure. Nevertheless the same $\Sigma=11$ GB can exhibit different structures depending on the deformation temperature. In the present case this might be also related to the two different translation states of the two adjacent grains in the $\Sigma=11$ GB.

\section{Acknowledgements.}

Prof. H.O.K. Kirchner is greatly acknowledged for fruitful discussions.

\section{References}

[1] PAPON A.M., PeTrT M., Scr. Met. 19 (1985) 391.

[2] d'ANTERroches C., Bourret A., Philos. Mag. A49 (1984) 71.

[3] KRIVANEK O., IsOdA S., KoBAYASHI K., Philos. Mag. A36 (1977) 931.

[4] Vaudin M., CunninghaM B., AST D., Scr. Met. 17 (1983) 191.

[5] Baillin X., Pelissier J., Bacmann J.J., JacQues A., George A., Philos. Mag. A56 (1987) 641.

[6] Jacques A., George A., Baillin W., Bacmann J.J., Philos. Mag. A55 (1987) 165.

[7] GeORge A., Jacoues A., Baillin X., Thibault-desseauX J., PUTauX J.L., Inst. Phys. Conf. Ser. 104 (1989) 349.

[8] Elkajbaji M., Thibault-Desseaux J., Philos. Mag. A58 (1988) 325.

[9] Baillin X., Pelissier J., JacQues A., GeOrge A., Philos. Mag. A61 (1990) 329.

[10] King A.H., ChEn F., Mater. Sci. Eng. 66 (1984) 227.

[11] Thibault J., PutauX J.L., Bourret A., KirChner H., J. Phys. France 50 (1989) 2525.

[12] Thibault J., ElkajBajI M., J. Phys. Colloq. France. 49 (1988) C5 283.

[13] Thibault-DesseauX J., PutauX J.L., Inst. Phys. Conf. 104 (1989) 1.

[14] Putaux J.L., Thibault-DesseauX J., J. Phys. Colloq. France 51 (1990) C1 323.

[15] Thibault J., PutauX J.L., ElkabaJ M., 12th Int. Cong. Elect. Micros. Seattle, 1990.

[16] HORNSTRA J., Physica 25 (1959) 409.

[17] Bourret A., BaCMANN J.J., Revue Phys. Appl. P. 22 (1987) 563.

[18] SuTton A., ViteK V., Philos. Trans. Roy. Soc. A309 (1983) 1. 